\title{
An extra copy of $p 53$ suppresses development of spontaneous Kras-driven but not radiation-induced cancer
}

\author{
Everett J. Moding, ${ }^{1}$ Hooney D. Min, ${ }^{2}$ Katherine D. Castle, ${ }^{1}$ Moiez Ali, ${ }^{1}$ Loretta Woodlief, ${ }^{2}$ \\ Nerissa Williams, ${ }^{2}$ Yan Ma, ${ }^{2}$ Yongbaek Kim, ${ }^{3}$ Chang-Lung Lee, ${ }^{2}$ and David G. Kirsch ${ }^{1,2}$ \\ 'Department of Pharmacology and Cancer Biology and 'Department of Radiation Oncology, Duke University, Durham, \\ North Carolina, USA. ${ }^{3}$ Laboratory of Veterinary Clinical Pathology, College of Veterinary Medicine, Seoul National \\ University, Seoul, South Korea.
}

The tumor suppressor $\mathrm{p} 53$ blocks tumor progression in multiple tumor types. Radiation-induced cancer following exposure to radiation therapy or space travel may also be regulated by $\mathrm{p} 53$ because p53 has been proposed to respond to DNA damage to suppress tumorigenesis. Here, we investigate the role of $\mathrm{p} 53$ in lung carcinogenesis and lymphomagenesis in $L A-1 \mathrm{Kras}^{\mathrm{G} 12 \mathrm{D}}$ mice with wild-type $p 53$ or an extra copy of $p 53$ (super $p 53$ ) exposed to fractionated total body irradiation with low linear energy transfer (low-LET) X-rays or high-LET iron ions and compared tumor formation in these mice with unirradiated controls. We found that an additional copy of $p 53$ suppressed both Kras-driven lung tumor and lymphoma development in the absence of radiation. However, an additional copy of $p 53$ did not affect lymphoma development following low- or high-LET radiation exposure and was unable to suppress radiation-induced expansion of thymocytes with mutated Kras. Moreover, radiation exposure increased lung tumor size in super $p 53$ but not wild-type $p 53$ mice. These results demonstrate that although $p 53$ suppresses the development of spontaneous tumors expressing $\mathrm{Kras}^{\mathrm{C} 12 \mathrm{D}}$, in the context of exposure to ionizing radiation, an extra copy of $p 53$ does not protect against radiation-induced lymphoma and may promote Kras ${ }^{\mathrm{G} 12 \mathrm{D}}$ mutant lung cancer.

Conflict of interest: The authors have declared that no conflict of interest exists.

Submitted: January 22, 2016

Accepted: June 2, 2016

Published: July 7, 2016

Reference information: JCI Insight. 2016;1(10):e86698. doi:10.1172/ji.insight.86698.

\section{Introduction}

p53 is a well-characterized tumor suppressor that has been shown to block both tumor initiation and progression in multiple tumor types (1-3). p53 responds to diverse cellular insults by triggering cell cycle arrest, senescence, or apoptosis depending on the tissue and type of stress (4). It has been proposed that the p53 response to DNA damage enables it to act as a guardian of the genome to suppress tumorigenesis (5). Interestingly, elephants, which have a lower-than-expected rate of cancer based on their large body size and long life span, were recently found to have at least 40 alleles of p53, and elephant lymphocytes underwent higher rates of radiation-induced apoptosis than human lymphocytes (6). It was proposed that cancer resistance in elephants is due to increased p53-mediated apoptosis following DNA damage. However, recent studies have separated the p53 transcriptional programs involved in the acute DNA damage response and tumor suppression, suggesting that the acute p53 response is dispensable for suppressing tumorigenesis (7-9). By temporarily knocking down p53 expression during radiation exposure in mice, we recently demonstrated that the acute $\mathrm{p} 53$ response to radiation promotes thymic lymphoma formation via a non-cell-autonomous mechanism (10). However, the effect of increasing p53 expression on radiation-induced carcinogenesis has not been investigated.

Development of second cancers is a devastating side effect of radiation therapy that is of particular concern for childhood cancer survivors (11). For example, the risk of second cancers following treatment for Hodgkin's lymphoma can approach $1 \%$ of patients per year following extended-field, high-dose radiation therapy (12). Risk of carcinogenesis following exposure to space radiation also limits the amount of time that astronauts can spend in deep space (13). Unlike terrestrial radiation, galactic cosmic rays in deep space are comprised of high charge and energy (HZE) particles that cause highly clustered DNA damage (14) and may be particularly mutagenic (15). Because humans are rarely exposed to HZE particles on earth, there is significant uncertainty about the risk of cancer following a mission into deep space (16). 
A

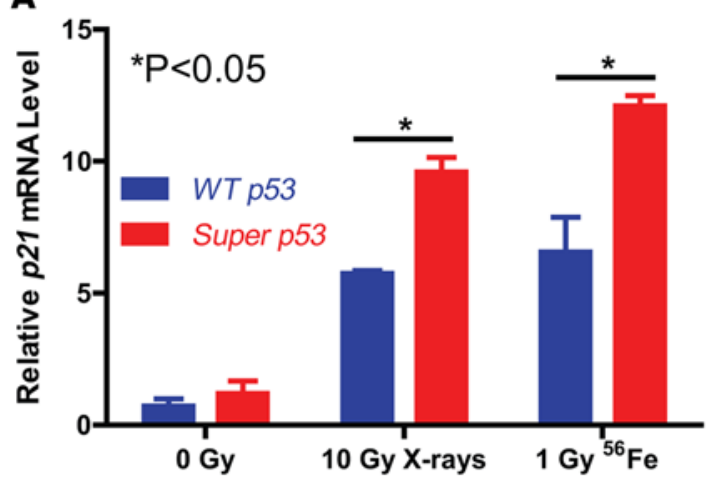

C

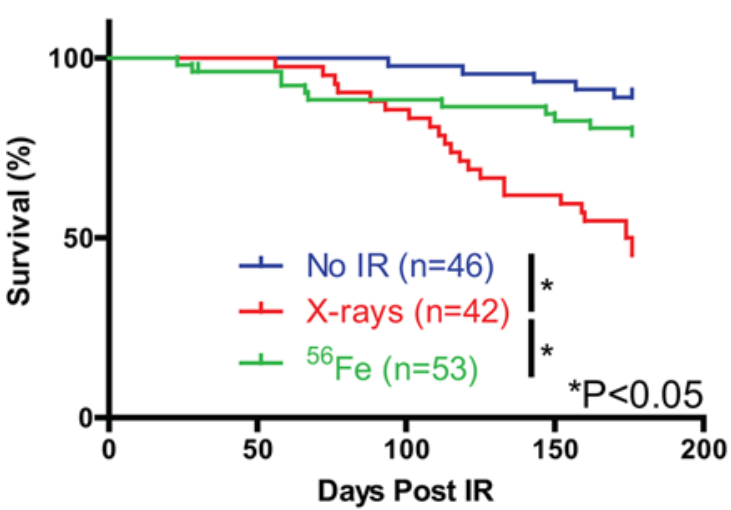

B

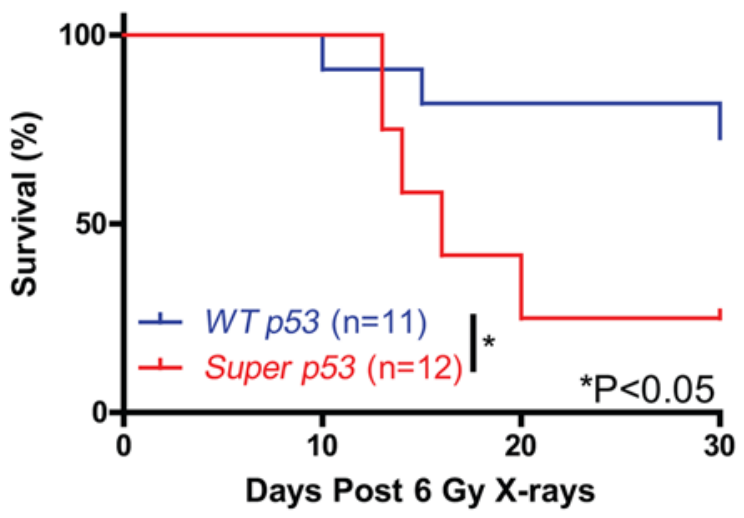

D LA-1; Super p53

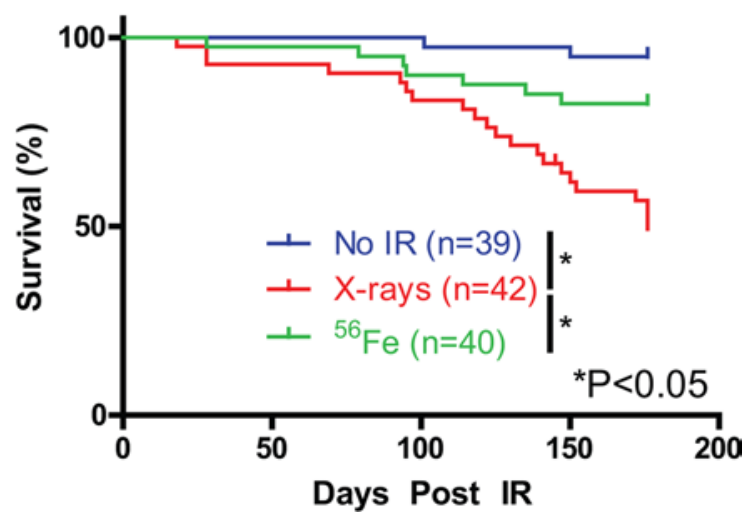

Figure 1. Exposure to fractionated X-ray radiation decreases survival of LA-1 mice. (A) Relative $p 21$ mRNA levels in the lungs of WT $p 53$ and super $p 53$ mice measured by qPCR 4 hours following exposure to no irradiation, 10 Gy X-rays, or 1 Gy ${ }^{56} \mathrm{Fe}(n=2)$. Data represent mean plus SEM. (B) Overall survival of WT p53 and super $p 53$ mice following exposure to a single dose of 6 Gy X-rays. Overall survival of (C) LA-1; WT p53 and (D) LA-1; super p53 mice following exposure to no irradiation (No IR), 5 daily fractions of 1.2 Gy X-rays, or 5 daily fractions of 0.2 Gy ${ }^{56} \mathrm{Fe} .{ }^{*} P<0.05$ by 2 -way ANOVA followed by Bonferroni's post-hoc test for $\mathbf{A}$ and Kaplan-Meyer analysis followed by log-rank test for $\mathbf{B}-\mathbf{D}$.

Although p53 plays a crucial role in carcinogenesis following X-ray irradiation $(17,18)$, the mechanisms of carcinogenesis following exposure to HZE particles with high linear energy transfer (high-LET) are not clear. Previous studies have demonstrated that the efficiency with which HZE particles induce cancer varies greatly across tumor types. For example, the relative biological effectiveness (RBE) of $1 \mathrm{GeV} /$ nucleon ${ }^{56} \mathrm{Fe}$ particles for acute myeloid leukemia (AML) is approximately 1, but the RBE for liver cancer appears to be closer to 50 (19). As a result, additional studies to determine the RBE for other tumor types are needed.

Lung cancer is the leading cause of cancer-related death worldwide (20). TP53, which encodes the transcription factor p53, and KRAS are the most commonly mutated genes in human lung adenocarcinoma, the most common histological subtype of lung cancer in humans (21). Oncogenic signals can activate p53 through numerous mechanisms, including increased expression of p19 ${ }^{\mathrm{ARF}}$, a tumor suppressor transcribed from the alternate reading frame of the INK4a/ARF locus (22). Although deletion of p53 has been shown to increase the progression of lung tumors $(23,24)$, the role of p53 in lung tumor initiation has not been thoroughly evaluated. Interestingly, p53 restoration in $K R A S$-mutant lung tumors has been shown to suppress progression of high-grade but not low-grade lung tumors due to the fact that KRAS mutation alone is insufficient to upregulate $\mathrm{p} 19^{\mathrm{ARF}}$ in normal lung (25-27).

$L A-1 \mathrm{Kras}^{G 12 D}(L A-1)$ mice undergo spontaneous recombination and activation of oncogenic Kras in somatic tissues (28). $L A-1$ mice develop low-grade lung adenomas with $100 \%$ penetrance and lymphomas at a lower rate. Exposure to fractionated low-LET and high-LET radiation has recently been shown to accelerate lung cancer progression in $L A-1$ mice (29). Deletion of p53 in $L A-1$ mice increases lung tumor grade (25), but the effect of p53 levels on tumor initiation has not been described. Super $p 53$ mice have been 
A

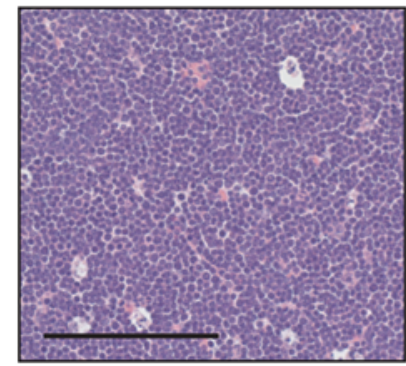

B

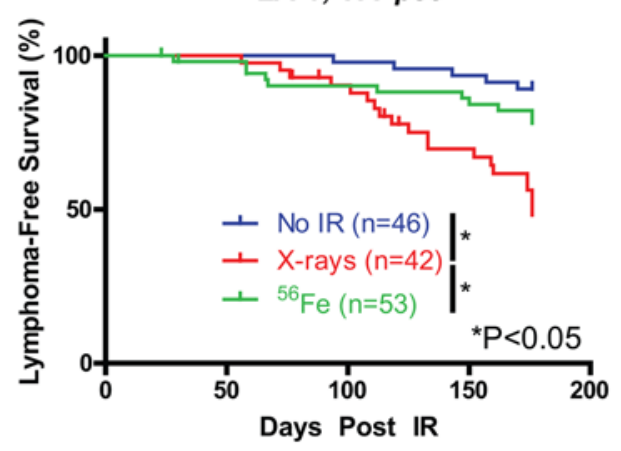

C

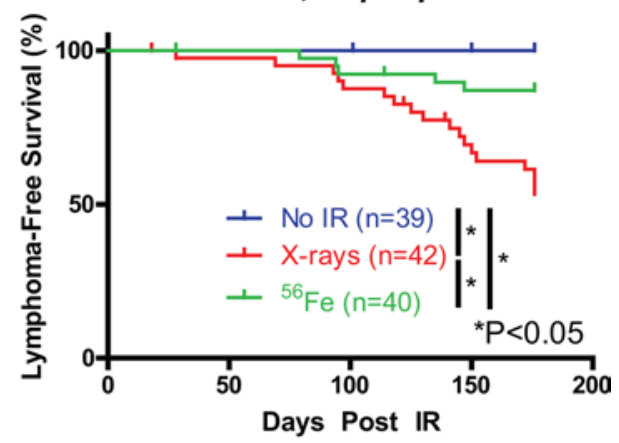

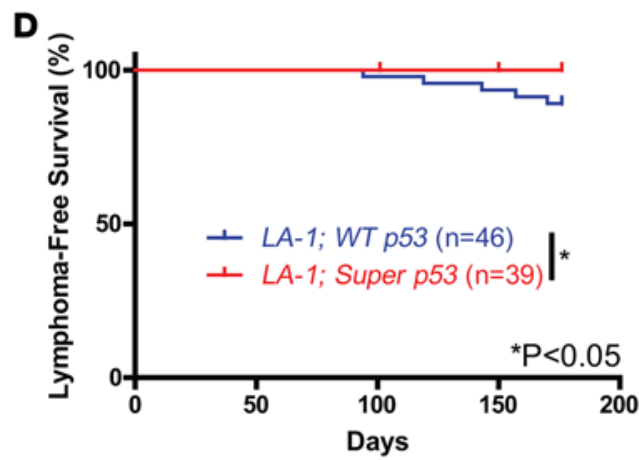

E

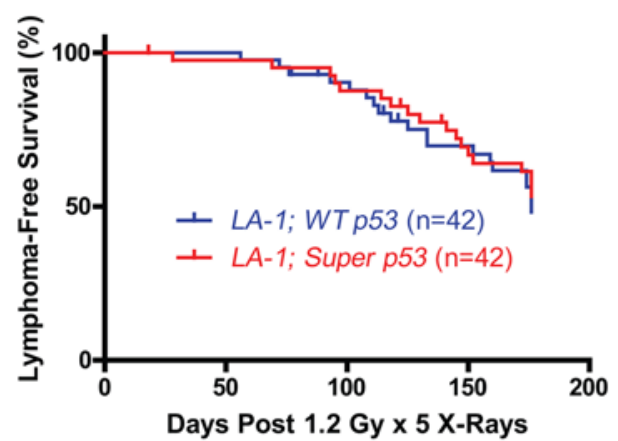

F

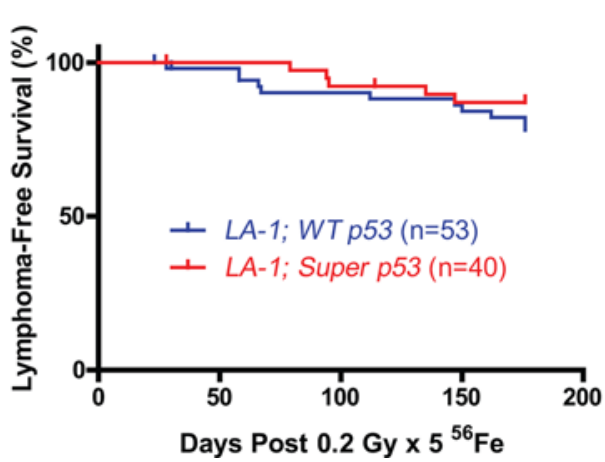

G

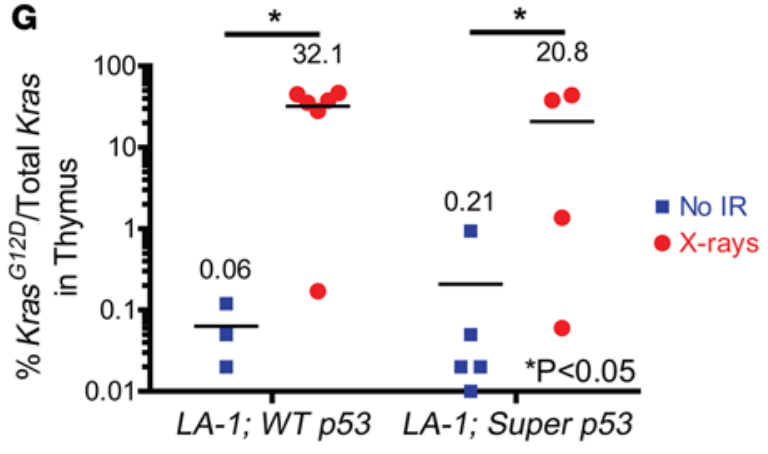

Figure 2. An extra copy of $p 53$ protects mice from spontaneous Kras ${ }^{6120}$-mutant lymphomas, but not radiation-induced $K_{\text {ras }}{ }^{6120}$-mutant lymphomas. (A) Example of a thymic lymphoma in an $L A-1$ mouse. Scale bar: $100 \mu \mathrm{m}$. Lymphoma-free survival in (B) $L A-1 ; W T p 53$ and (C) $L A-1$; super $p 53$ mice following exposure to no irradiation (No IR), 5 daily fractions of 1.2 Gy X-rays, or 5 daily fractions of 0.2 Gy ${ }^{56} \mathrm{Fe}$. Lymphoma-free survival in $L A-1 ; W T p 53$ and $L A-1$; super $p 53$ mice following exposure to (D) no irradiation, (E) 5 daily fractions of 1.2 Gy X-rays, or (F) 5 daily fractions of 0.2 Gy ${ }^{56} \mathrm{Fe}$. (C) Percentage of the recombined Kras ${ }^{C 12 D}$ allele in thymocytes from $L A-1 ; W T p 53$ and $L A-1$; super $p 53$ mice 70 days after no irradiation or 1.2 Gy $X$-rays $(n=3-6)$. Bars represent means. Data from $\mathbf{B}$ and $\mathbf{C}$ are repeated in $\mathbf{D}-\mathbf{F}$ for additional comparisons. ${ }^{*} P<0.05$ by Kaplan-Meyer analysis followed by log-rank test for $\mathbf{B}-\mathbf{F}$ and 2 -way ANOVA followed by Bonferroni's post-hoc test for $\mathbf{G}$.

genetically engineered to carry an extra copy of p53 and are resistant to cancer development (30). Because the majority of studies investigating the role of p53 in carcinogenesis have utilized p53 mutation or deletion rather than overexpression (31), super $p 53$ mice represent a unique gain-of-function approach to study the role of $\mathrm{p} 53$ in radiation-induced cancer.

In this study, we exposed LA-1 mice with 2 (wild-type) or 3 (super p53) copies of $p 53$ to fractionated low-LET radiation to model radiation therapy for cancer patients and high-LET radiation to simulate the chronic exposure to space radiation that astronauts would experience on a mission to deep space. Although an extra copy of $p 53$ suppressed the spontaneous development of $\mathrm{Kras}^{\mathrm{G} 12 \mathrm{D}}$-mutant lymphomas and lung tumors in the absence of radiation, super $p 53$ mice were not protected from the development of radiationinduced lymphomas. Moreover, both low-LET and high-LET radiation exposure increased lung tumor size in super p53, but not wild-type p53 mice. These experiments demonstrate that p53 suppresses tumorigenesis following the spontaneous expression of oncogenic Kras, but p53 can promote cancer development in the context of exposure to ionizing radiation. 

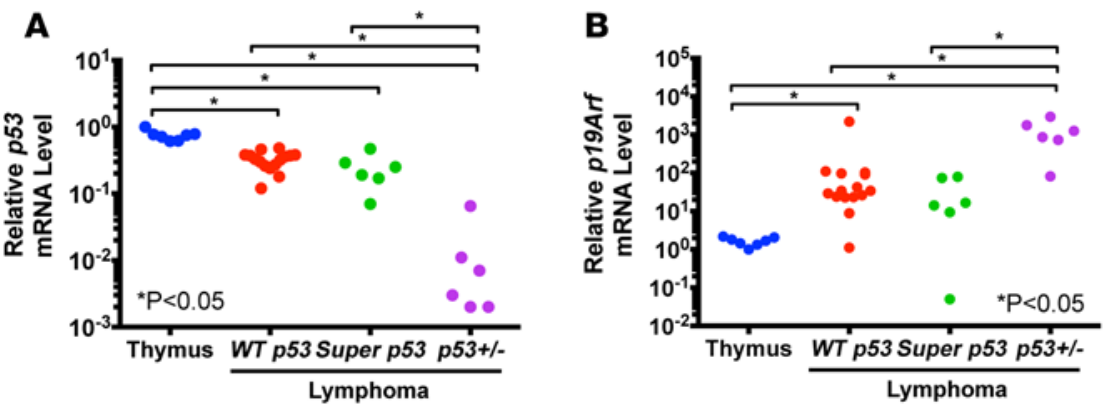
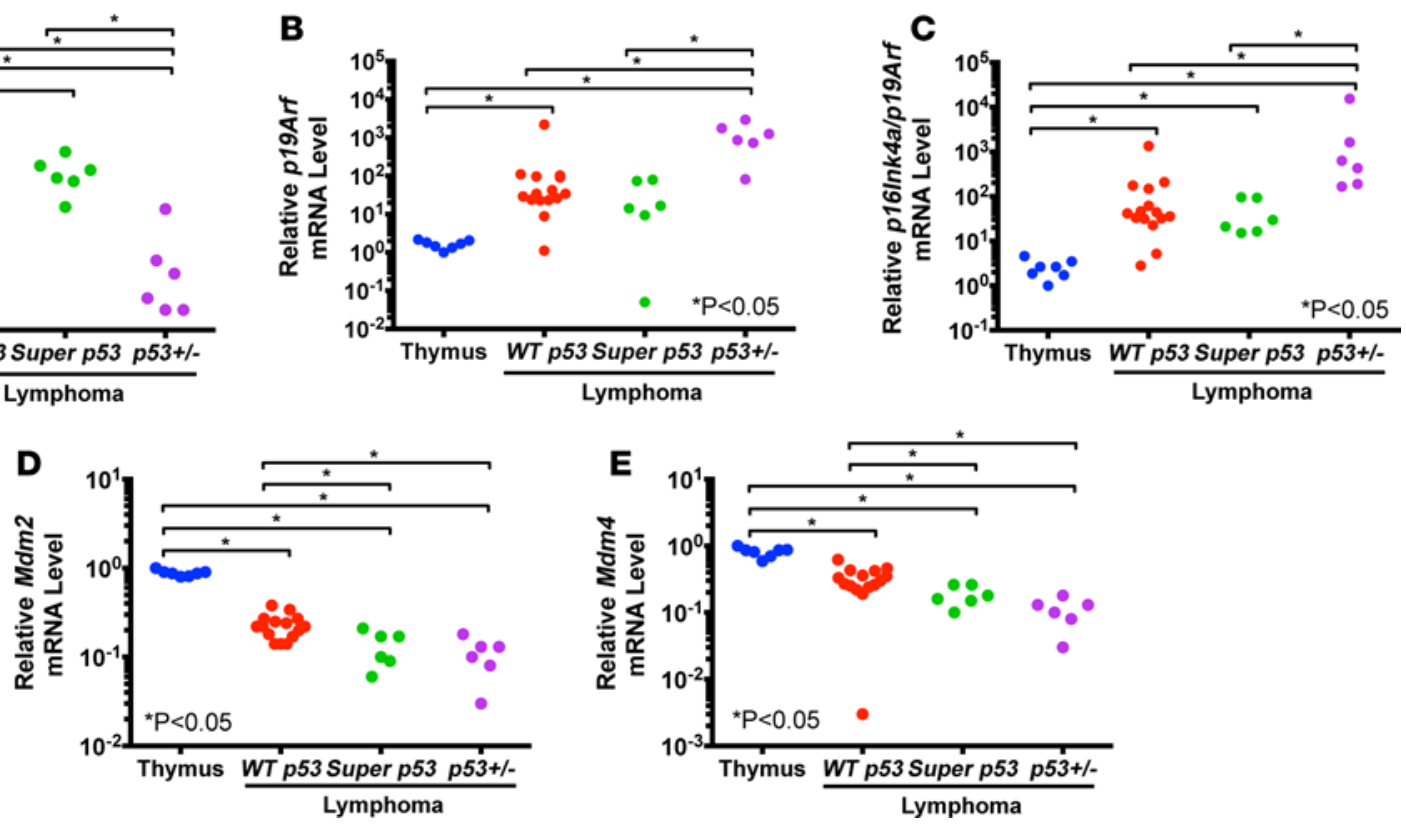

Figure 3. p19Arf expression is increased while expression of $\mathbf{M d m 2}$ and $\mathbf{M d m} \mathbf{4}$ is decreased in radiation-induced lymphomas. Relative mRNA levels of (A) p53, (B) p19Arf, (C) combined p16Ink4a and p19Arf, (D) Mdm2, and (E) Mdm4 in wild-type p53 thymuses (Thymus) and radiation-induced lymphomas from WT $p 53$, super $p 53$, and $p 53^{+/-}$mice $(n=6-15)$. Each symbol represents 1 mouse. ${ }^{*} P<0.05$ by Kruskal-Wallis test followed by Mann-Whitney test.

\section{Results}

An extra copy of p53 suppresses spontaneous Kras-mutant, but not radiation-induced lymphomas. To determine if super 553 mice have increased p53 activation after low-LET and high-LET radiation, we exposed wildtype (WT) p53 and super 553 mice to $10 \mathrm{~Gy}$ X-rays (low-LET) or $1 \mathrm{~Gy}$ iron ions (high-LET) and harvested the lungs for quantitative RT-PCR. The level of $p 53$ mRNA was significantly greater in super p53 mice in the presence and absence of radiation (Supplemental Figure 1A; supplemental material available online with this article; doi:10.1172/jci.insight.86698DS1). Furthermore, super p53 mice had significantly more induction of the p53 transcriptional targets $p 21$ and $M d m 2$ than WT $p 53$ mice following both low-LET and high-LET radiation exposure (Figure 1A and Supplemental Figure 1B). In contrast, the induction of p53 targets Puma and Phlda3 depended on the type of radiation exposure (Supplemental Figure 1, C and D). To examine if acute radiation toxicity is increased in super $p 53$ mice, we exposed $W T p 53$ and super $p 53$ mice to total body irradiation with 6 Gy X-rays to induce the hematopoietic acute radiation syndrome. Super p53 mice had significantly reduced survival compared to WT p53 mice (Figure 1B), demonstrating that an extra copy of p53 sensitizes mice to the hematopoietic acute radiation syndrome.

To investigate the role of $\mathrm{p} 53$ in low-LET and high-LET radiation-induced cancer, we exposed $L A-1$; WT p53 and LA-1; super p53 mice to no irradiation or 5 daily fractions of $1.2 \mathrm{~Gy} \mathrm{X}$-rays or $0.2 \mathrm{~Gy}{ }^{56} \mathrm{Fe}$ and monitored them for up to 6 months for tumor formation (Supplemental Table 1). We chose 5 daily fractions of $0.2 \mathrm{~Gy}$ iron ions because this dose and fractionation has been shown to increase the incidence of invasive lung adenocarcinoma in $L A$ - 1 mice (29). An X-ray dose of $1.2 \mathrm{~Gy}$ was chosen because this was the maximum dose that could be delivered for 5 consecutive days without causing bone-marrow failure and the hematopoietic acute radiation syndrome. Low-LET radiation exposure significantly decreased overall survival of both $L A-1$; WT p53 and LA-1; super p53 mice compared to mice exposed to high-LET radiation or unirradiated controls (Figure 1, C and D).

A full necropsy determined that the majority of $L A-1$ mice that died following low-LET radiation exposure developed thymic lymphomas (Figure 2A). Low-LET radiation exposure significantly increased lymphoma incidence in both $L A-1 ; W T$ p53 and $L A-1$; super $p 53$ mice compared to mice exposed to either no radiation or high-LET radiation (Figure 2, B and C). LA-1; super $p 53$ mice had significantly fewer lymphomas than $L A-1$; WT 553 mice in the absence of radiation (Figure 2D). However, there was no significant difference in lymphoma latency between $L A-1 ; W T$ p53 and $L A-1$; super $p 53$ mice following exposure to radiation (Figure $2, \mathrm{E}$ and $\mathrm{F}$ ). We did not observe any lymphomas with $p 53$ mutations for either genotype in 

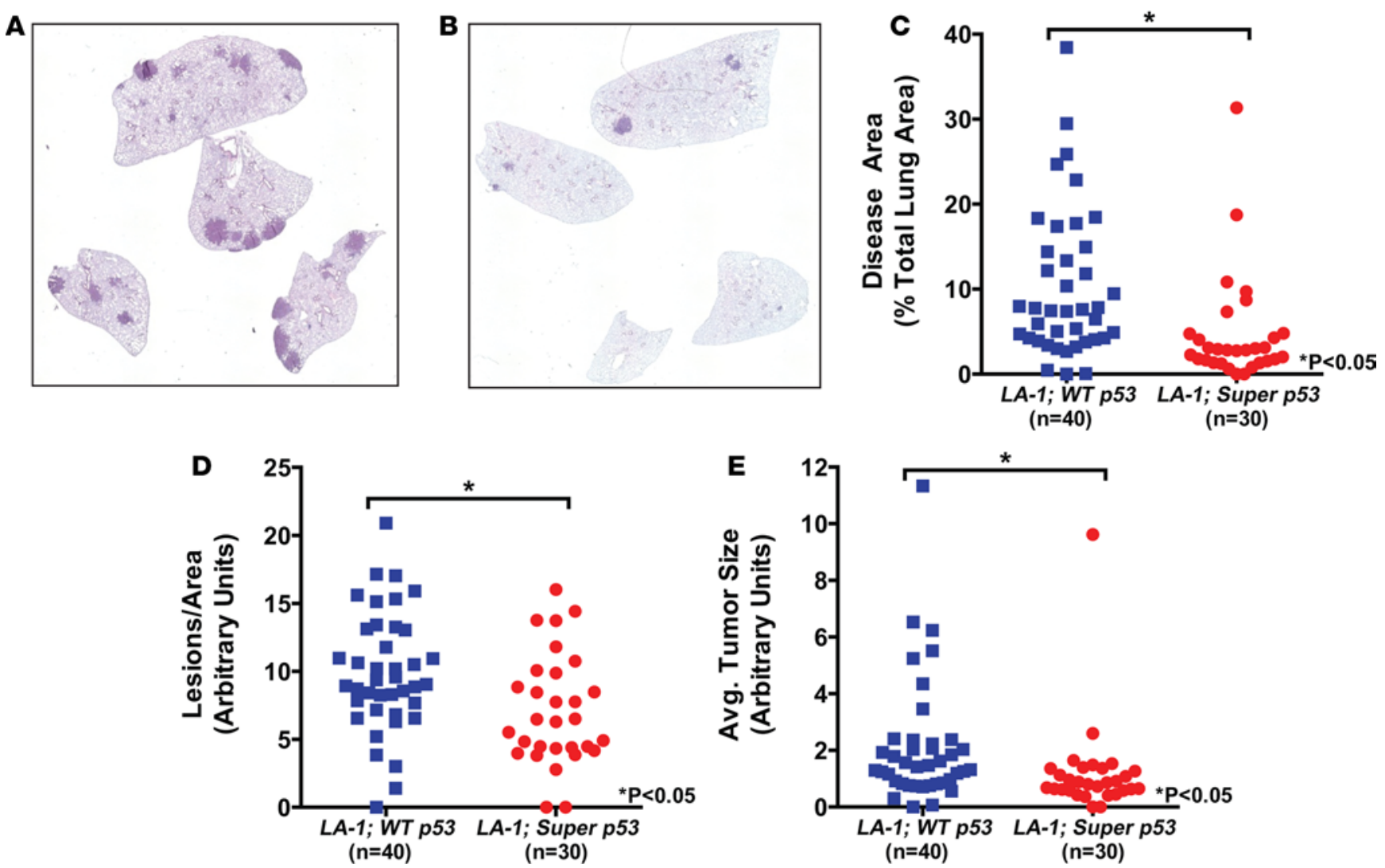

Figure 4. An extra copy of $\mathbf{p} 53$ blocks Kras ${ }^{\mathrm{C} 120}$-mutant lung tumor development. Representative hematoxylin and eosin-stained sections of unirradiated lungs from (A) an $L A-1$; WT p53 mouse and (B) an $L A-1$; super $p 53$ mouse. Images were obtained at $\times 25$ magnification. Quantification of (C) tumor burden, (D) normalized tumor number, and (E) average tumor size in unirradiated LA-1; WT p53 and LA-1; super p53 mice. Each symbol represents 1 mouse. ${ }^{*} P<0.05$ by Mann-Whitney test.

the presence or absence of radiation (Supplemental Table 2). To examine whether an extra copy of p53 suppresses the expansion of Kras-mutant thymocytes, we quantified the recombined $\mathrm{Kras}^{G 12 D}$ allele in thymuses via droplet digital PCR in $L A-1$; WT 553 and $L A-1$; super $p 53$ mice 70 days after no irradiation or irradiation with X-rays. X-ray exposure increased the frequency of the $\mathrm{Kras}^{G 12 D}$ allele, but there was no difference in $K_{r a s}^{G 12 D}$ frequency between genotypes (Figure 2G). To further test whether super $p 53$ mice are protected from radiation-induced lymphomas, we repeated the low-LET radiation experiment in $W T$ p53 and super p53 mice without the $L A-1$ allele. There was no difference in overall survival or lymphoma-free survival between $W T$ p 53 and super 53 mice exposed to 5 daily fractions of $1.2 \mathrm{~Gy}$ X-rays (Supplemental Figure 2). These results suggest that although $p 53$ can suppress the development of spontaneous Kras-driven lymphomas, an extra copy of $p 53$ does not suppress radiation-induced lymphomagenesis.

To better understand the mechanisms by which lymphomas evade p53-mediated tumor suppression, we examined the mRNA expression of p53, p19Avf, Mdm2, and Mdm4 in WT p53 mouse thymuses, and in radiation-induced lymphomas from $W T$ p 53 and super $p 53$ mice (Figure 3 ). We included radiation-induced lymphomas from $p 53^{+/-}$mice as an additional control because they have previously been shown to undergo p53 loss of heterozygosity (17). Expression of p53, Mdm2, and Mdm4 was significantly reduced in lymphomas of all genotypes compared with $W T$ p53 thymuses. In contrast, p19Arf expression was significantly increased in lymphomas with no significant difference between lymphomas in WT p53 and super p53 mice.

An extra copy of p53 suppresses development of spontaneous Kras-mutant lung adenomas. To analyze lung tumor development in LA-1; WT p53 and LA-1; super p53 mice, we euthanized the mice that survived to 6 months following radiation along with age-matched unirradiated controls and collected their lungs for histology (Figure 4, A and B). Because lung tumor burden increases with age in LA-1 mice (28), mice that died prior to this time point were not included in the analysis. First, we investigated whether an extra copy of $p 53$ altered lung tumor burden in the absence of radiation. LA-1; super p53 mice had a 

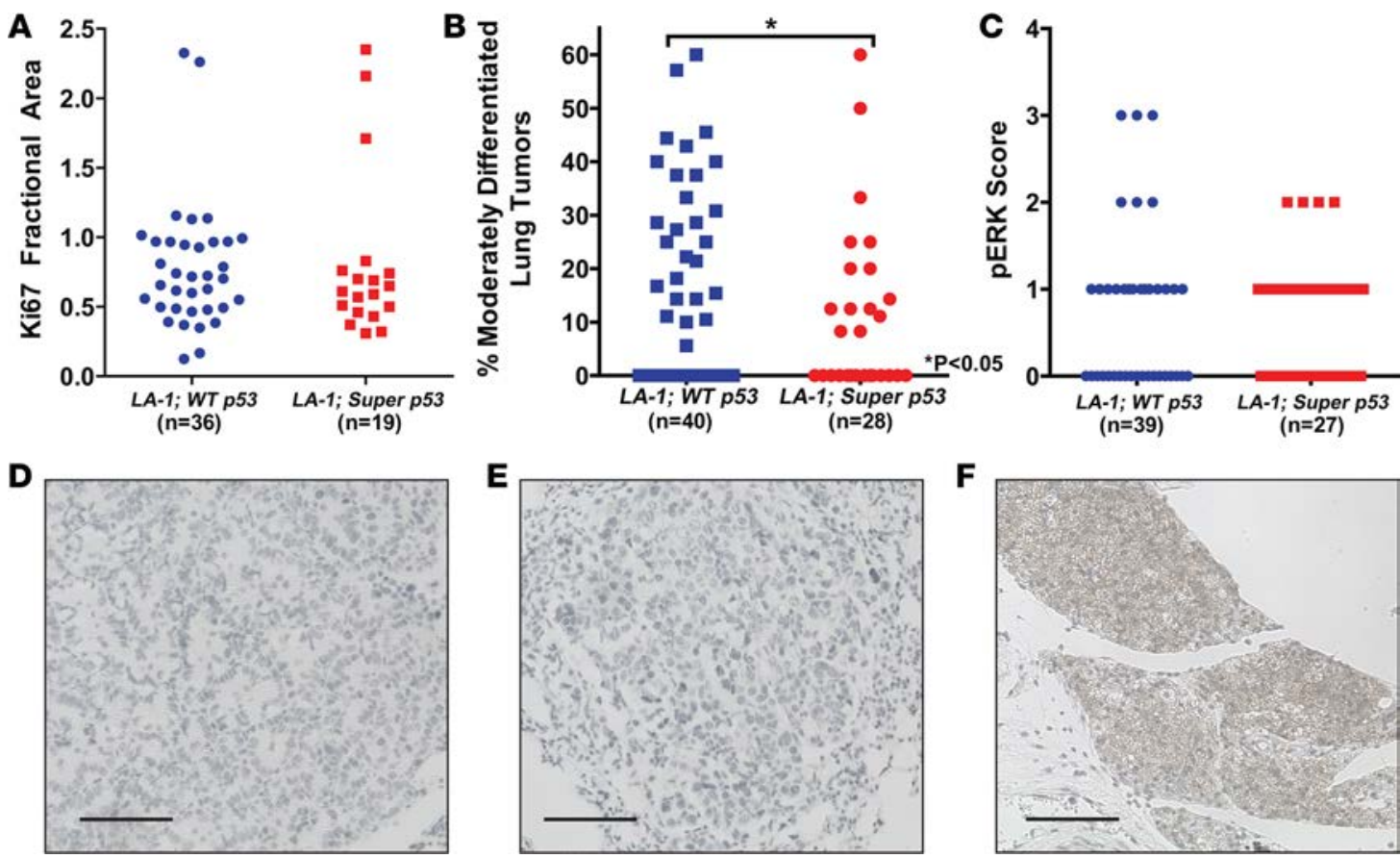

Figure 5. An extra copy of $\mathbf{p} 53$ decreases tumor grade but does not affect lung adenoma growth. (A) Quantification of the cellular proliferation marker Ki67 in lung tumors from $L A-1 ; W T p 53$ and $L A-1$; super $p 53$ mice. Each symbol represents 1 tumor. (B) Quantification of tumor grade in $L A-1 ; W T p 53$ and LA-1; super $p 53$ mice. Each symbol represents 1 mouse. (C) Quantification of phospho-ERK (pERK) staining in lung tumors from LA-1; WT p53 and LA-1; super $p 53$ mice. Each symbol represents 1 tumor. Representative sections of lung tumors from (D) an $L A-1 ; W T p 53$ mouse and (E) an $L A-1 ;$ super $p 53$ mouse stained for p19ARF. (F) p19ARF staining of a $p 53$-null lung tumor as a positive control. Scale bars: $100 \mu \mathrm{m} .{ }^{*} P<0.05$ by Mann-Whitney test.

significantly reduced lung tumor burden compared to $L A-1 ; W T$ p53 mice (Figure 4C). To determine if the reduction in tumor burden was due to a reduction in tumor initiation or tumor growth, we quantified lung tumor size and number. LA-1; super 553 mice had significantly fewer lung tumors and smaller lung tumors than $L A-1 ; W T$ p 53 mice (Figure 4, D and E).

To investigate whether $p 53$ alters lung adenoma proliferation, we stained the lungs from $5 L A-1 ; W T$ p53 and LA-1; super p53 mice for the proliferation marker Ki67 and the mitosis marker phospho-histone $\mathrm{H} 3$ (pHH3). An extra copy of p53 did not affect lung adenoma Ki67 staining (Figure 5A) or pHH3 staining (Supplemental Figure 3), suggesting that the reduction in lung tumor size in $L A$-1; super p53 mice was due to delayed tumor initiation rather than differences in tumor growth. Because p53 blocks progression of high-grade but not low-grade lung tumors $(25,26)$, we compared tumor grade in $L A-1 ; W T$; 53 and $L A-1$; super p53 mice. An extra copy of p53 significantly decreased the percentage of moderately differentiated lung tumors in $L A-1$ mice (Figure $5 \mathrm{~B}$ ). No poorly differentiated lung tumors were observed in any of the mice in this study.

Kras signals through multiple pathways to increase cellular proliferation and survival. Activation of the mitogen-activated protein kinase (MAPK) pathway has been associated with advanced non-small-cell lung cancer in humans (32). Consistent with our finding that an extra copy of p53 blocks lung tumor initiation but not proliferation, there was no significant difference in the level of phospho-ERK (pERK) staining between lung adenomas in $L A-1$; WT p 53 and LA-1; super $p 53$ mice (Figure $5 \mathrm{C}$ ).

The differential effect of p53 on suppression of low- and high-grade lung tumors has been linked to differences in p19ARF expression (25-27). Lung adenomas from LA-1; WT p53 and LA-1; super p53 mice did not stain for p19ARF (Figure 5, D-F). These results suggest that an extra copy of $p 53$ does not block proliferation of lung adenomas in $L A-1$ mice because Kras activation is insufficient to upregulate $\mathrm{p} 19^{\mathrm{ARF}}$ in the lung.

Exposure to high-LET radiation increases lung tumor size in LA-1; super p53 mice. To investigate effects of radiation exposure on lung tumor development in $L A-1$ mice, we quantified lung tumors in $L A-1 ; W T$ p53 and $L A$-1; super $p 53$ mice following radiation exposure. Neither low-LET nor high-LET radiation exposure affected lung tumor burden in $L A-1 ; W T$ p 53 mice (Figure 6, A-C). However, exposure to either low-LET or high-LET radiation increased lung tumor burden by increasing lung tumor size in $L A-1$; super p53 mice 

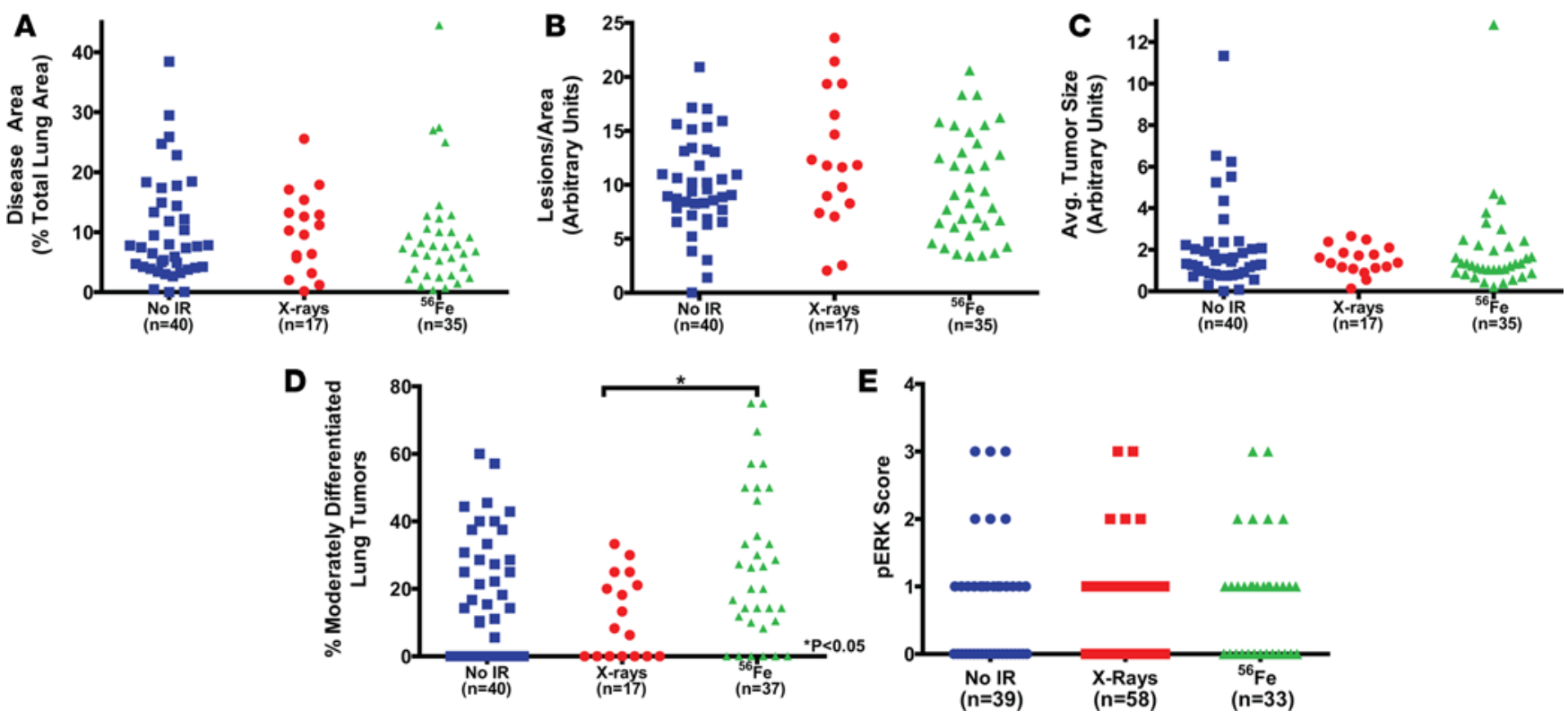

Figure 6. Exposure to radiation does not affect lung tumor burden in LA-1; WT p53 mice. Quantification of (A) tumor burden, (B) normalized tumor number, (C) average tumor size, (D) tumor grade, and (E) phospho-ERK (pERK) staining in LA-1; WT p53 mice 6 months following exposure to no irradiation (No IR), 5 daily fractions of 1.2 Gy X-rays, or 5 daily fractions of 0.2 Gy ${ }^{56} \mathrm{Fe}$. Each symbol represents 1 mouse (A-D) or 1 tumor (E). No-irradiation data repeated from Figures 4 and 5 for additional comparisons. ${ }^{*} P<0.05$ by Kruskal-Wallis test followed by Mann-Whitney test.

(Figure $7, \mathrm{~A}-\mathrm{C}$ ). These results suggest that an extra copy of $p 53$ increases the susceptibility of $L A-1$ mice to radiation-induced lung cancer growth.

We also analyzed lung tumor grade following radiation exposure to determine if radiation affects lung adenoma differentiation. High-LET radiation exposure significantly increased the percentage of moderately differentiated lung tumors compared to low-LET radiation exposure in $L A-1$; WT $p 53$ mice (Figure 6D). However, compared to unirradiated $L A-1 ; W T p 53$ mice, mice exposed to ${ }^{56} \mathrm{Fe}$ did not show a statistically significant increase in lung tumor grade at 6 months. Although there was a trend towards increased tumor grade in LA-1; super $p 53$ mice following high-LET radiation exposure, there was not a statistically significant change (Figure 7D). There was no significant change in the level of pERK staining as a result of radiation exposure for either $L A-1$; WT $p 53$ (Figure 6E) or LA-1; super $p 53$ mice (Figure 7E). Finally, consistent with the lymphomas that developed in these mice, we did not observe any $p 53$ mutations in the lung tumors examined in this study (Supplemental Table 2).

\section{Discussion}

In this study we demonstrate, to our knowledge for the first time, that although p53 can block spontaneous tumorigenesis driven by oncogenic mutation of Kras in both the thymus and the lung, an extra copy of p53 does not block development of radiation-induced $\mathrm{Kras}^{G 12 D}$-mutant lymphomas and increases the impact of radiation on $\mathrm{Kras}^{112 D}$-mutant lung tumor development. These results demonstrate the variable and contextdependent role of $\mathrm{p} 53$ in tumor suppression and highlight potential approaches to suppress carcinogenesis by modulating the p53 tumor suppressor pathway.

Although numerous studies have demonstrated that p53 can block progression of high-grade lung tumors $(23,24)$, to our knowledge, this is the first study to find that p53 can block initiation of lung adenomas. Consistent with previous studies $(25,26)$, an extra copy of p53 did not affect proliferation of low-grade lung adenomas as measured by Ki67, pHH3, and pERK staining. This appears to result from failure of mutant Kras expression to activate p19 ${ }^{\mathrm{ARF}}$. In addition, although p53 does not block progression of lung adenomas, intact $\mathrm{p} 53$ signaling appears to be essential for blocking initiation of lung tumorigenesis. Because increased Kras signaling and elevated p19 $9^{\mathrm{ARF}}$ trigger $\mathrm{p} 53$ tumor suppression in high-grade lung tumors, interventions could potentially trigger $\mathrm{p} 53$ in low-grade lung adenomas by elevating p $19^{\mathrm{ARF}}$ expression.

Recent studies have demonstrated that the acute $\mathrm{p} 53$ response is not essential to block radiation-induced lymphoma formation $(7,8)$. Furthermore, we recently demonstrated that $\mathrm{p} 53$ acts during radiation to promote 

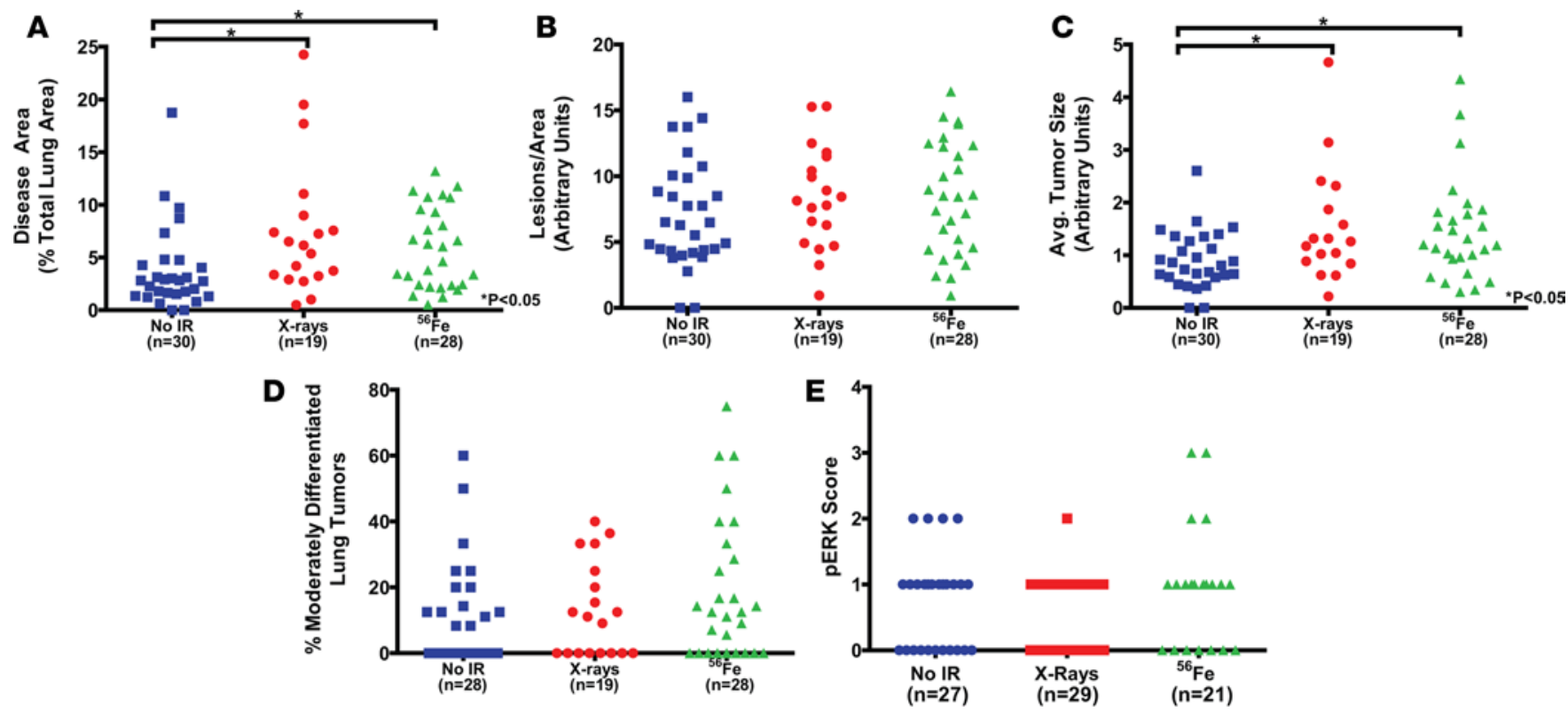

Figure 7. Radiation exposure increases lung tumor burden in LA-1; super p53 mice. Quantification of (A) tumor burden, (B) normalized tumor number, (C) average tumor size, (D) tumor grade, and (E) phospho-ERK (pERK) staining in LA-1; super p53 mice 6 months following exposure to no irradiation (No IR), 5 daily fractions of 1.2 Gy X-rays, or 5 daily fractions of 0.2 Gy ${ }^{56} \mathrm{Fe}$. Each symbol represents 1 mouse (A-D) or 1 tumor (E). No-irradiation data repeated from Figures 4 and 5 for additional comparisons. ${ }^{*} P<0.05$ by Kruskal-Wallis test followed by Mann-Whitney test.

lymphoma formation (10). Our observation that super $p 53$ mice are protected from spontaneous Kras-driven lymphoma formation, but not radiation-induced $K r a s^{G 12 D}$-mutant lymphomagenesis, further demonstrates that the role of p53 in tumor suppression is dependent on the specific stimulus that activates p53: oncogenic stress that acts in a cell-autonomous manner versus ionizing radiation that activates p53 not only in the tumorinitiating cell, but also in other cells. We observed that an extra copy of $p 53$ suppressed lymphomagenesis in the absence of radiation but also increased the hematopoietic acute radiation syndrome. Therefore, it appears that any tumor-suppressive, cell-autonomous effects of having an additional copy of $p 53$ are cancelled out by the tumor-promoting non-cell-autonomous acute effects of $\mathrm{p} 53$ during radiation.

Our finding that $L A-1$; super $p 53$ mice but not $L A-1$; WT $p 53$ mice are susceptible to radiation-induced increases in lung tumor size suggests that the acute $p 53$ response can promote radiation-induced solid tumor growth. It is unclear if the acute $p 53$ response in super $p 53$ mice is acting in lung cancer cells or in the tumor microenvironment, and additional experiments in other model systems will be necessary to make this distinction. Taken together with our recent work (10), these results provide further support for utilizing inhibitors of p53 during radiation exposure to not only ameliorate acute radiation injury, but also to prevent radiation-induced tumorigenesis. Notably, using low-dose arsenic to inhibit p53 during administration of chemotherapy was recently shown to reduce hematologic toxicity in a clinical trial (33). Although the increase in lung tumor size following radiation in super p53 mice was relatively small, our findings raise the possibility that drugs that increase p53 signaling during radiation exposure, such as $\mathrm{Mdm} 2$ inhibitors, could promote cancer progression. In addition, our results support the idea that additional copies of $p 53$ mediate cancer resistance in elephants (6). However, our results suggest a model in which decreased tumor development results from the increased response of p53 to oncogenic stress rather than the acute p53 response to DNA damage.

We explored how an extra copy of $p 53$ regulates lymphoma development in different contexts by quantifying the recombined $\mathrm{Kras}^{G 12 D}$ allele in the thymuses of mice after irradiation and in unirradiated controls. Similar to our prior results (10), radiation exposure led to the expansion of cells with the recombined Kras $^{G 12 D}$ allele in the thymus. This does not appear to be due to radiation-induced recombination of the $L A-1$ allele, because we previously did not observe an increase in the number of cells with the recombined Kras $^{G 12 D}$ allele 8 days after radiation (10).

We observed that low-LET radiation significantly increased the incidence of lymphomas in both genotypes of mice compared to no irradiation or high-LET irradiation. High-LET radiation exposure increased 
lymphomagenesis in both $L A-1$; WT p53 and LA-1; super p53 mice, but the difference was not statistically significant in $W T$ p53 mice. In this study, the dose of low-LET radiation was 6 times greater than the dose of high-LET radiation. This demonstrates that the RBE for $600 \mathrm{MeV} /$ nucleon iron ions is significantly less than 6 compared with $320 \mathrm{kVp}$ X-rays, suggesting that the effect of high-LET radiation on lymphomagenesis may be more similar to hematologic malignancies such as AML than solid cancers (19). Our finding that radiation exposure did not affect lung tumor number may be a result of limitations of the $L A-1$ model. At the time of radiation, $L A-1$ mice had already started developing multiple lung adenomas. It is possible that other genetically engineered mouse models in which oncogene activation can be temporally controlled may provide further insights into the effect of radiation on lung tumor initiation. High-LET radiation increased lung tumor grade in the $L A-1$ model compared with low-LET radiation, but this was not significant compared to unirradiated controls. At 6 months after radiation exposure, most of the lung tumors were welldifferentiated adenomas, and we observed no poorly differentiated, invasive lung tumors. It is possible that changes in tumor grade would be greater at later time points following radiation exposure (29).

In summary, we found that an extra copy of $p 53$ blocks development of spontaneous Kras-driven lymphomas and lung cancers, but not radiation-induced lymphomas. These results demonstrate the contextdependent role of $\mathrm{p} 53$-mediated tumor suppression and further suggest that the acute $\mathrm{p} 53$ response to DNA damage may promote tumorigenesis rather than enable p53 to act as a guardian of the genome. Therefore, increasing p53 signaling during radiation therapy may increase the risk of second tumors.

\section{Methods}

Mice. $L A-1$ and super p53 Tg mice have been described previously $(28,30)$. $L A-1$ mice were provided by Jerry Shay (University of Texas Southwestern Medical Center, Dallas, Texas, USA) and Tyler Jacks (Massachusetts Institute of Technology, Cambridge, Massachusetts, USA), and super $p 53$ mice were provided by Manuel Serrano (Spanish National Cancer Research Centre, Madrid, Spain). All mice were maintained on a C57BL/ 6 background and age-matched, littermate controls were utilized for experiments. For gene expression analysis, $W T$ p 53 and p53-deficient radiation-induced lymphomas as well as $W T p 53$ thymus tissues were collected from mice as described previously (10).

Radiation exposure. For all experiments, male and female mice were exposed to total body irradiation between 6 and 12 weeks of age. X-ray irradiations were performed at Duke University using an X-RAD 320 Biological Irradiator (Precision X-ray). Mice were placed $50 \mathrm{~cm}$ from the radiation source and were irradiated with a dose rate of $200 \mathrm{cGy} / \mathrm{min}$ using $320 \mathrm{kVp}, 12.5 \mathrm{~mA}$ X-rays and a filter consisting of $2.5-\mathrm{mm} \mathrm{Al}$ and $0.1-\mathrm{mm} \mathrm{Cu}$. The dose rate was measured with an ion chamber by members of the Radiation Safety Division at Duke University. For ${ }^{56} \mathrm{Fe}$ irradiations, mice were shipped to Brookhaven National Laboratory where they were irradiated at the NASA Space Radiation Laboratory with 600 $\mathrm{MeV} /$ nucleon ${ }^{56} \mathrm{Fe}$ ions at a dose rate of $20 \mathrm{cGy} / \mathrm{min}$. Following ${ }^{56} \mathrm{Fe}$ irradiation, the mice were returned to Duke University where they were monitored for tumor development. To account for shipping effects, unirradiated control mice were included in each shipment to Brookhaven National Laboratory. We did not observe any difference in tumor formation between unirradiated mice maintained at Duke University and unirradiated mice shipped to Brookhaven National Laboratory (Supplemental Figure 4), so these groups were combined for all analyses.

Quantitative RT-PCR analysis. Total RNA was extracted from whole lungs using TRIzol reagent (Invitrogen), and reverse transcription was performed with the iScript cDNA Synthesis Kit (Bio-Rad). Quantitative reverse transcription-polymerase chain reaction (qRT-PCR) was performed using a Taqman Universal PCR Master Mix and TaqMan Gene Expression Assay Mix (both Applied Biosystems) for Cdkn1a ( 211$)$ (Mm00432448_m1), Bbc3 (Puma) (Mm00519268_m1), Mdm2 (Mm01233136_m1), Mdm4 (Mm00484944_ m1), Phlda3 (Mm00449846_m1), Cdkn2a (p16Ink4a + p19Arf) (Mm0049449_m1), Cdkn2a (p19Arf) (Mm01257348_m1), p53 (Mm01731290_g1), Gapdh (Mm99999915_g1), or Hprt (Mm0446968_m1). Hprt or Gapdh was used as an internal control to correct for the concentration of cDNA in different samples. Each experiment was performed with 3 replicates from each sample, and the results were averaged.

Tumor analysis. Following irradiation, mice were monitored twice daily for 6 months or until they became moribund. At necropsy, the lungs were inflated via intratracheal infusion and were collected along with thymic lymphomas for histological analysis. Lung lobes were separated and tissue specimens were fixed in $10 \%$ neutralized formalin overnight and preserved in $70 \%$ ethanol until paraffin embedding. Five-micron sections taken $300 \mu \mathrm{m}$ into the lungs were used for hematoxylin and eosin staining 
or immunohistochemistry. Pictures of hematoxylin and eosin-stained slides were acquired with a Leica DFC340 FX microscope using Leica Suite software (Leica Microsystems). Lung and tumor areas were quantified by an observer blinded to treatment and genotype using ImageJ (NIH) for tumor and lung thresholding. Tumors were graded by an observer blinded to treatment and genotype in consultation with a veterinary pathologist as described previously (10). Well-differentiated (low-grade) tumors had regular to slightly irregular nuclei. Moderately differentiated (intermediated-grade) tumors had enlarged, pleomorphic nuclei with prominent nucleoli and nuclear molding. Poorly differentiated (high-grade) tumors demonstrated aberrant mitoses, tumor giant cells, and desmoplasia.

Immunohistochemistry. Lung sections were deparaffinized with xylene and rehydrated with a graded series of ethanol and water washes prior to antigen retrieval using Antigen Unmasking Solution (Vector Laboratories) according to the manufacturer's instructions. The primary antibodies used were Ki67 (1:250, BD Pharmingen, catalog 550609), pERK1/2 (1:500, Cell Signaling, catalog 4370), and p19 ARF (1:500, Novus Biologicals, catalog NB200-174). Biotinylated secondary antibodies were detected using the Vectastain Elite ABC Reagent (Vector Laboratories) and Sigmafast diaminobenzidine tablets (Sigma-Aldrich). Slides were counterstained with Mayer's hematoxylin (Sigma-Aldrich). Quantification of Ki67 fractional area was performed using ImageJ. pERK scoring was determined by the percentage of the tumor that stained positive. Score 0, 0\%; Score 1, 0\%-25\%; Score 2, 25\%-50\%; Score 3, > 50\%.

Quantification of the recombined Kras ${ }^{G 12 D}$ allele. Quantification of the recombined Kras ${ }^{G 12 D}$ allele in the thymus of $L A-1$ mice was performed as described previously (10). Briefly, genomic DNA was extracted from thymuses using the DNeasy Blood \& Tissue Kit (QIAGEN). Then, PCR was performed to amplify exon 1 of the Kras allele, and the 3.5-kb amplicon was purified using the QIAquick PCR Purification Kit (QIAGEN). Droplet digital PCR was performed on the purified amplicon using the RainDrop Digital PCR System (RainDance Technologies) with Taqman MGB probes (Applied Biosystems) designed to specifically detect the WT Kras allele and ras $^{G 12 D}$ allele. Data were analyzed using the RainDrop Analyst data analysis software (RainDance Technologies).

p53 sequencing. Exome sequencing was performed to assess for mutations in the DNA binding domain of $p 53$ in the lung tumors and lymphomas. Genomic DNA was purified from formalin-fixed, paraffin-embedded lymphomas and lung tumors using the QIAamp DNA FFPE Tissue Kit (QIAGEN). PCR amplification of exon 4 was performed using the forward primer 5'-CTACATAGCAAGTTGGAGGCCAG-3' and reverse primer 5'-AAGAGGCATTGAAAGGTCACACG-3'. Exons 5 and 6 were amplified using the forward primer 5'-GGAGGAAGAAGGAAAGGTCCCAG-3' and reverse primer 5'-ACCGGACTCAGCGTCTCTATTTC-3'. Amplicons were purified using the QIAquick PCR Purification Kit (QIAGEN) and sequenced at the Duke University DNA Analysis Facility using the same primers used for PCR amplification. Sequencing results were analyzed for mutations using CLC Sequence Viewer (CLC bio).

Statistics. qRT-PCR of p53 targets in lungs and droplet digital PCR data were analyzed by 2-way ANOVA to examine the interaction between genotypes and treatments followed by Bonferroni's post-hoc tests for pairwise comparisons of individual genotypes. Non-normally distributed data were log transformed before applying statistical tests. For survival studies, Kaplan-Meier analysis was performed followed by the log-rank test for statistical significance. For lung-tumor analysis and qRT-PCR of thymuses and lymphomas, 2-tailed Mann-Whitney tests were performed to compare the distributions of 2 groups. Kruskal-Wallis tests followed by Mann-Whitney tests were performed to compare more than 2 groups. Significance was assumed at $P<0.05$. All calculations were performed using Prism 5 (GraphPad).

Study approval. All animal studies were performed in accordance with protocols approved by the Duke University and Brookhaven National Laboratory Institutional Animal Care and Use Committees.

\section{Author contributions}

EJM, CLL, and DGK conceived and designed the experiments. EJM, HDM, CLL, KDC, and MA performed the experiments. LW and NW participated in the animal studies. YM processed histological specimens. YK reviewed the histopathology. EJM and DGK analyzed the data and wrote the manuscript.

\section{Acknowledgments}

These studies were supported by NASA NSCOR NNX11AC60G (to D.G. Kirsch), NCI F30 CA177220 (to E.J. Moding), and MEST NRF 2013R1A2A2A01068237 (to Y. Kim). We thank Jerry Shay and Tyler 
Jacks for providing the $L A-1$ mice on a C57BL/ 6 background and Manuel Serrano for providing the super $p 53$ mice. We thank the staff at the NASA Space Radiation Laboratory for helping to irradiate mice. We thank Lixia Luo and the staff of the Brookhaven Animal Facility and Duke Laboratory Animal Resources for caring for the mice, and Chris Miller for assisting with droplet digital PCR experiments.

Address correspondence to: David G. Kirsch, Duke University Medical Center, Box 91006, Durham, North Carolina 27708, USA. Phone: 919.681.8605; E-mail: david.kirsch@duke.edu.

1. Young NP, Crowley D, Jacks T. Uncoupling cancer mutations reveals critical timing of p53 loss in sarcomagenesis. Cancer Res. 2011;71(11):4040-4047.

2. Jonkers J, Meuwissen R, van der Gulden H, Peterse H, van der Valk M, Berns A. Synergistic tumor suppressor activity of BRCA2 and p53 in a conditional mouse model for breast cancer. Nat Genet. 2001;29(4):418-425.

3. Schwitalla S, et al. Loss of p53 in enterocytes generates an inflammatory microenvironment enabling invasion and lymph node metastasis of carcinogen-induced colorectal tumors. Cancer Cell. 2013;23(1):93-106.

4. Lee CL, Blum JM, Kirsch DG. Role of p53 in regulating tissue response to radiation by mechanisms independent of apoptosis. Transl Cancer Res. 2013;2(5):412-421.

5. Lane DP. Cancer. p53, guardian of the genome. Nature. 1992;358(6381):15-16

6. Abegglen LM, et al. Potential mechanisms for cancer resistance in elephants and comparative cellular response to DNA damage in humans. JAMA. 2015;314(17):1850-1860.

7. Brady CA, et al. Distinct p53 transcriptional programs dictate acute DNA-damage responses and tumor suppression. Cell. 2011;145(4):571-583.

8. Christophorou MA, Ringshausen I, Finch AJ, Swigart LB, Evan GI. The pathological response to DNA damage does not contribute to p53-mediated tumour suppression. Nature. 2006;443(7108):214-217.

9. Li T, et al. Tumor suppression in the absence of p53-mediated cell-cycle arrest, apoptosis, and senescence. Cell. 2012;149(6):1269-1283.

10. Lee CL, et al. Acute DNA damage activates the tumour suppressor p53 to promote radiation-induced lymphoma. Nat Commun. $2015 ; 6: 8477$.

11. Bhatia S, Sklar C. Second cancers in survivors of childhood cancer. Nat Rev Cancer. 2002;2(2):124-132.

12. Bhatia S, et al. Breast cancer and other second neoplasms after childhood Hodgkin's disease. N Engl J Med. 1996;334(12):745-751.

13. Durante M, Cucinotta FA. Heavy ion carcinogenesis and human space exploration. Nat Rev Cancer. 2008;8(6):465-472.

14. Sutherland BM, Bennett PV, Sidorkina O, Laval J. Clustered DNA damages induced in isolated DNA and in human cells by low doses of ionizing radiation. Proc Natl Acad Sci U S A. 2000;97(1):103-108.

15. Kiefer J. Mutagenic effects of heavy charged particles. J Radiat Res. 2002;43(suppl):S21-S25.

16. Cucinotta FA, Chappell LJ. Updates to astronaut radiation limits: radiation risks for never-smokers. Radiat Res. 2011;176(1):102-114.

17. Kemp CJ, Wheldon T, Balmain A. p53-deficient mice are extremely susceptible to radiation-induced tumorigenesis. Nat Genet. 1994;8(1):66-69.

18. Lee JM, Abrahamson JL, Kandel R, Donehower LA, Bernstein A. Susceptibility to radiation-carcinogenesis and accumulation of chromosomal breakage in p53 deficient mice. Oncogene. 1994;9(12):3731-3736.

19. Weil MM, et al. Incidence of acute myeloid leukemia and hepatocellular carcinoma in mice irradiated with $1 \mathrm{GeV} /$ nucleon $(56)$ Fe ions. Radiat Res. 2009;172(2):213-219.

20. Herbst RS, Heymach JV, Lippman SM. Lung cancer. N Engl J Med. 2008;359(13):1367-1380.

21. Ding L, et al. Somatic mutations affect key pathways in lung adenocarcinoma. Nature. 2008;455(7216):1069-1075.

22. Kamijo T, et al. Tumor suppression at the mouse INK4a locus mediated by the alternative reading frame product p19ARF. Cell. 1997;91(5):649-659.

23. Jackson EL, et al. The differential effects of mutant p53 alleles on advanced murine lung cancer. Cancer Res. 2005;65(22):10280-10288

24. Dankort D, Filenova E, Collado M, Serrano M, Jones K, McMahon M. A new mouse model to explore the initiation, progression, and therapy of BRAFV600E-induced lung tumors. Genes Dev. 2007;21(4):379-384.

25. Feldser DM, et al. Stage-specific sensitivity to p53 restoration during lung cancer progression. Nature. 2010;468(7323):572-575.

26. Junttila MR, et al. Selective activation of p53-mediated tumour suppression in high-grade tumours. Nature. 2010;468(7323):567-571.

27. Young NP, Jacks T. Tissue-specific p19Arf regulation dictates the response to oncogenic K-ras. Proc Natl Acad Sci U S A. 2010;107(22):10184-10189.

28. Johnson L, et al. Somatic activation of the K-ras oncogene causes early onset lung cancer in mice. Nature. 2001;410(6832):1111-1116.

29. Delgado O, et al. Radiation-enhanced lung cancer progression in a transgenic mouse model of lung cancer is predictive of outcomes in human lung and breast cancer. Clin Cancer Res. 2014;20(6):1610-1622.

30. Garcia-Cao I, et al. "Super p53" mice exhibit enhanced DNA damage response, are tumor resistant and age normally. EMBO J. 2002;21(22):6225-6235.

31. Kenzelmann Broz D, Attardi LD. In vivo analysis of p53 tumor suppressor function using genetically engineered mouse models. Carcinogenesis. 2010;31(8):1311-1318.

32. Vicent $\mathrm{S}$, et al. ERK1/2 is activated in non-small-cell lung cancer and associated with advanced tumours. Br J Cancer. 2004;90(5):1047-1052.

33. Ha CS, et al. p53-based strategy to reduce hematological toxicity of chemotherapy: A proof of principle study. Mol Oncol. 2016;10(1):148-156 